\title{
Comparative Theoretical Analysis of Distance Estimation in Visible Light Positioning Systems
}

\author{
Musa Furkan Keskin, Student Member, IEEE, and Sinan Gezici, Senior Member, IEEE
}

\begin{abstract}
In this paper, theoretical limits and estimators are studied for synchronous and asynchronous visible light positioning (VLP) systems. Specifically, the Cramér-Rao lower bounds (CRLBs) and maximum likelihood estimators are investigated for distance estimation based on time-of-arrival (TOA) and/or received signal strength (RSS) parameters. Hybrid TOA/RSS-based distance estimation is proposed for VLP systems, and its CRLB is compared analytically against the CRLBs of TOA-based and RSSbased distance estimation. In addition, to investigate the effects of sampling, asymptotic performance results are obtained under sampling rate limitations as the noise variance converges to zero. A modified hybrid TOA/RSS-based distance estimator is proposed to provide performance improvements in the presence of sampling rate limitations. Numerical examples are presented to illustrate the theoretical results.
\end{abstract}

Index Terms-Cramér-Rao lower bound, estimation, Lambertian pattern, positioning, visible light.

\section{INTRODUCTION}

$\mathbf{R}$ ECENTLY, light emitting diode (LED) based visible light communication (VLC) has attracted significant attention [1]-[4]. VLC systems can provide both illumination and high speed data transmission for indoor environments. In addition to communications, LEDs can also be utilized for positioning [5]-[10]. Since multipath effects are not significant in line-ofsight (LOS) visible light channels, accurate positioning can be performed via visible light positioning (VLP) systems. High accuracy provided by VLP systems can facilitate various applications and services such as robot navigation, asset tracking, and location specific advertisement [2], [5].

In VLP systems, various types of parameters such as received signal strength (RSS), time-of-arrival (TOA), time-differenceof-arrival (TDOA), and angle-of-arrival (AOA) can be employed for position estimation. In RSS based systems, the position of a VLC receiver is estimated based on RSS measurements between the VLC receiver and a number of LED transmitters [8], [9], [11]-[14]. Unlike in radio frequency (RF) based systems, the RSS parameter can provide very accurate position related information in VLP systems since the channel attenuation factor does not fluctuate significantly in LOS visible light channels.

Manuscript received August 25, 2015; revised October 9, 2015 and November 22, 2015; accepted November 24, 2015. Date of publication November 25, 2015; date of current version February 5, 2016. This work was supported in part by the Distinguished Young Scientist Award of the Turkish Academy of Sciences (TUBA-GEBIP 2013).

The authors are with the Department of Electrical and Electronics Engineering, Bilkent University, Ankara 06800, Turkey (e-mail: keskin@ee. bilkent.edu.tr; gezici@ee.bilkent.edu.tr).

Color versions of one or more of the figures in this paper are available online at http://ieeexplore.ieee.org.

Digital Object Identifier 10.1109/JLT.2015.2504130
In [8], a complete VLP system based on RSS measurements and trilateration is implemented and the achieved sub-meter accuracy is compared against other positioning systems. In [9], Kalman and particle filtering are employed for RSS based position tracking in VLP systems. The study in [11] utilizes a single LED transmitter and multiple optical receivers for position estimation, where the position of the receiver unit is determined based on RSS measurements at multiple receivers. In [13], an RSS based VLP system is designed and a multiaccess protocol is implemented. The proposed system can guarantee decimeter level accuracy in almost all scenarios in the presence and absence of direct sunlight exposure. A carrier allocation VLC system is proposed in [14] for RSS based positioning and experiments are performed to illustrate its centimeter level average positioning accuracy. The studies in [6] and [15] consider the use of the time delay parameter for positioning. In particular, [6] investigates the theoretical limits on TOA estimation for visible light systems. In [15], TDOAs are calculated at a VLC receiver based on signals from three LEDs and two-dimensional (2-D) position estimation is performed based on TDOAs. As another alternative, the AOA parameter can be utilized for localization in VLP systems [10], [16], [17]. For example, the study in [10] considers a multi-element VLC system and exploits the narrow field of view of LEDs to extract position related information from connectivity conditions. Based on a least-squares estimator and Kalman filtering, average positioning accuracy on the order of $0.2 \mathrm{~m}$ is reported.

Although there exist many studies on VLP systems, theoretical limits on estimation accuracy have been considered very rarely [6], [7]. Theoretical limits for estimation present useful performance bounds on mean-squared errors (MSEs) of estimators and provide important guidelines for system design. In [6], the Cramér-Rao lower bound (CRLB) is presented for distance (or, TOA) estimation in a synchronous VLC system. The effects of various system parameters, such as source optical power, center frequency, and the area of the photo detector, are investigated. Simulation results indicate centimeter level accuracy limits for typical system parameters. The study in [7] derives the CRLB for distance estimation based on the RSS parameter, and investigates the dependence of the CRLB expression on system parameters such as LED configuration, transmitter height, and the signal bandwidth. Again, CRLBs on the order of centimeters are observed for typical system parameters.

In this study, a generic signal model, which covers TOA based [6] and RSS based [7] distance estimation as special cases, is considered, and theoretical limits and estimators are derived. In particular, the CRLBs and maximum likelihood estimators (MLEs) are investigated for both synchronous and asynchronous 
scenarios and in the presence and absence of a relation between distance and channel attenuation factor. In this way, in addition to TOA based and RSS based distance estimation, hybrid TOA/RSS based distance estimation is introduced for VLP systems, and theoretical links and comparisons are provided between the current study and those in the literature [6], [7]. Also, via the CRLB expressions, the accuracy limits for TOA based, RSS based, and hybrid TOA/RSS based distance estimation are compared analytically. Furthermore, asymptotic results are obtained for the MLEs under sampling rate limitations, and a modified hybrid estimator is proposed to perform accurate distance estimation in practical scenarios. The main contributions and novelty of the paper can be summarized as follows:

- The hybrid RSS/TOA based distance estimation is proposed for VLP systems for the first time. In addition, the CRLB and the MLE corresponding to the hybrid RSS/TOA based distance estimation are derived, which have not been available in the literature ${ }^{1}$.

- Analytical expressions are derived for the ratios between the CRLBs for the TOA based, RSS based, and hybrid TOA/RSS based distance estimation. In particular, it is shown that the CRLB for the hybrid TOA/RSS based estimation converges to that of the TOA based distance estimation for $\beta \gg c / x$, and to that of the RSS based distance estimation for $\beta \ll c / x$, where $\beta$ is the effective bandwidth of the transmitted signal, $x$ is the distance between the LED transmitter and the VLC receiver, and $c$ is the speed of light.

- Effects of sampling rate limitations on the TOA based, RSS based, and hybrid TOA/RSS based MLEs are characterized via asymptotic MSE expressions as the noise variance converges to zero.

- To provide performance improvements in the presence of sampling rate limitations, a modified hybrid TOA/RSS based estimator is proposed based on the hybrid TOA/RSS based MLE.

In addition, slightly more general CRLB expressions than those in [6] and [7] are presented for the TOA based and RSS based distance estimation, and the conditions under which the CRLB expressions in [6] and [7] arise are specified. Furthermore, comparisons among different approaches are provided in terms of theoretical estimation accuracy and robustness to sampling rate limitations. Numerical examples are provided to investigate the theoretical results.

The remainder of the paper is organized as follows: The system model is introduced and the parameters are defined in Section II. The CRLBs and the MLEs are derived for synchronous and asynchronous scenarios in Section III, and comparisons are presented among the CRLBs in various cases. In Section IV, the asymptotic MSEs are derived for the MLEs when the noise variance goes to zero, and the modified hybrid TOA/RSS based distance estimator is proposed. Numerical examples are presented in Section V, followed by the concluding remarks in Section VII.

\footnotetext{
${ }^{1}$ The hybrid RSS/TOA based estimation and the corresponding CRLB and MLE expressions in RF positioning systems [18]-[21] are different from those in this study due to the distinct characteristics of the visible light channel.
}

\section{SYSTEM MODEL}

In an indoor VLP system, LED transmitters are commonly located on the ceiling of a room, and a VLC receiver is located on an object on the floor. Based on the signals received from the LED transmitters (which have known positions), the VLC receiver can estimate its distance (range) to each LED transmitter and determine its position based on distance estimates. The aim in this study is to investigate the fundamental limits on distance estimation.

Consider an LED transmitter at location $\boldsymbol{l}_{t} \in \mathbb{R}^{3}$ and a VLC receiver at location $\boldsymbol{l}_{r} \in \mathbb{R}^{3}$ in an LOS scenario. The distance between the LED transmitter and the VLC receiver is represented by $x$, which is given by $x=\left\|\boldsymbol{l}_{r}-\boldsymbol{l}_{t}\right\|_{2}$. The received signal at the VLC receiver is expressed as [6]

$$
r(t)=\alpha R_{p} s(t-\tau)+n(t)
$$

for $t \in\left[T_{1}, T_{2}\right]$, where $T_{1}$ and $T_{2}$ specify the observation interval, $\alpha$ is the attenuation factor of the optical channel $(\alpha>0)$, $R_{p}$ is the responsivity of the photo detector, $s(t)$ is the transmitted signal which is nonzero over an interval of $\left[0, T_{s}\right], \tau$ is the TOA, and $n(t)$ is zero-mean additive white Gaussian noise with a spectral density level of $\sigma^{2}$. It is assumed that $R_{p}$ and $s(t)$ are known by the VLC receiver. Also, the TOA parameter is modeled as

$$
\tau=\frac{x}{c}+\Delta
$$

where $x$ is the distance between the LED transmitter and the VLC receiver, $c$ is the speed of light, and $\Delta$ denotes the time offset between the clocks of the LED transmitter and the VLC receiver. For a synchronous system, $\Delta=0$, whereas for an asynchronous system, $\Delta$ is modeled as a deterministic unknown parameter. It is assumed that coarse acquisition is performed so that the signal component in (1) resides completely in the observation interval $\left[T_{1}, T_{2}\right]$.

The channel attenuation factor $\alpha$ in (1) is modeled as

$$
\alpha=\frac{m+1}{2 \pi} \cos ^{m}(\phi) \cos (\theta) \frac{A_{R}}{x^{2}}
$$

where $m$ is the Lambertian order, $A_{R}$ is the area of the photo detector at the VLC receiver, $\phi$ is the irradiation angle, and $\theta$ is the incidence angle [6], [8]. For compactness of analytical expressions, it is assumed, similarly to [6], [7], [11], that the LED transmitter is pointing downwards (which is commonly the case) and the photo detector at the VLC receiver is pointing upwards such that $\phi=\theta$ and $\cos (\phi)=\cos (\theta)=h / x$, where $h$ denotes the height of the LED transmitter relative to the VLC receiver ${ }^{2}$. In addition, as in [6], [7], [9], [11], it is assumed that the height of the VLC receiver is known; that is, possible positions of the VLC receiver are confined to a 2-D plane. This assumption holds in various practical scenarios; e.g., when the VLC receiver is attached to a cart or a robot that is tracked via a VLP system as VLC receivers have fixed and known heights in such applications (e.g., [5, Fig. 3]). Under these assumptions,

\footnotetext{
${ }^{2}$ It is straightforward to extend the theoretical bounds in this study to the cases with arbitrary transmitter and receiver orientations. However, it is not performed as the expressions become lengthy and inconvenient.
} 
(3) becomes

$$
\alpha=\frac{m+1}{2 \pi}\left(\frac{h}{x}\right)^{m+1} \frac{A_{R}}{x^{2}} \triangleq \gamma x^{-m-3}
$$

where $\gamma \triangleq(m+1) h^{m+1} A_{R} /(2 \pi)$ is a known constant ${ }^{3}$.

\section{CRLBS AND ML EsTIMATORS}

In order to calculate the CRLB, the log-likelihood function corresponding to the received signal model in (1) is specified as follows [22], [23]:

$$
\Lambda(\boldsymbol{\varphi})=k-\frac{1}{2 \sigma^{2}} \int_{T_{1}}^{T_{2}}\left(r(t)-\alpha R_{p} s(t-\tau)\right)^{2} d t
$$

where $\varphi$ denotes the set of unknown parameters including $x$ and other nuisance parameters, if any, depending on the considered scenario (as discussed below), and $k$ represents a normalizing constant that is a function of $\sigma$ and does not depend on the unknown parameter(s). The CRLB is obtained based on the inverse of the Fisher information matrix (FIM) for $\varphi$, which can be calculated from the log-likelihood function in (5) as [24]

$$
\mathbf{J}(\boldsymbol{\varphi})=\mathbb{E}\left\{\left(\nabla_{\boldsymbol{\varphi}} \Lambda(\boldsymbol{\varphi})\right)\left(\nabla_{\boldsymbol{\varphi}} \Lambda(\boldsymbol{\varphi})\right)^{T}\right\}
$$

where $\nabla_{\varphi}$ represents the gradient operator with respect to $\varphi$. From the FIM in (6), the CRLB on the covariance matrix of any unbiased estimator $\hat{\varphi}$ of $\varphi$ can be calculated as follows:

$$
\mathbb{E}\left\{(\hat{\boldsymbol{\varphi}}-\boldsymbol{\varphi})(\hat{\boldsymbol{\varphi}}-\boldsymbol{\varphi})^{T}\right\} \succeq \mathbf{J}(\boldsymbol{\varphi})^{-1}
$$

where $\boldsymbol{A} \succeq \boldsymbol{B}$ means that $\boldsymbol{A}-\boldsymbol{B}$ is positive semidefinite [24].

In the following, the CRLBs and MLEs are derived for different cases.

\section{A. Case 1: Synchronous System}

Firstly, the following assumptions are considered: $(i)$ the LED transmitter and the VLC receiver are synchronized (i.e., $\Delta=0$ in (2)) and ( $i i)$ the relation of channel attenuation factor $\alpha$ to distance $x$ is unknown; i.e., a relation as in (4) is not available. The latter is a common assumption in RF based distance estimation systems (e.g., [25]) since the channel coefficient fluctuates significantly due to multipath effects (fading). However, in visible light systems, the channel attenuation factor can accurately be related to distance, especially in LOS scenarios, and this relation can be used to improve the accuracy of distance estimation, as will be discussed later in this section. The main aims behind studying distance estimation in the absence of the relation between $\alpha$ and $x$ are to provide a benchmark for analyzing the effects of this relation, and to investigate the previous results in the literature [6].

In the presence of synchronization and in the absence of a relation between the channel attenuation factor and distance,

\footnotetext{
${ }^{3}$ The assumption of a known height is required for unambiguous estimation of distance based on an RSS measurement (cf., (4)).
}

the ML estimator [24] can be obtained from (5) as follows:

$$
\begin{aligned}
\hat{x}_{\mathrm{ML}, \mathrm{TOA}} & =\underset{\varphi}{\arg \max } \frac{-1}{2 \sigma^{2}} \int_{T_{1}}^{T_{2}}\left(r(t)-\alpha R_{p} s(t-\tau)\right)^{2} d t \\
& =\underset{x}{\arg \max } \int_{T_{1}}^{T_{2}} r(t) s\left(t-\frac{x}{c}\right) d t
\end{aligned}
$$

where the final expression is obtained due to the facts that $\alpha>0$ and the TOA parameter in (2) becomes $\tau=x / c$ for a synchronous system.

For the CRLB derivation in this scenario, it is first assumed that the channel attenuation factor $\alpha$ is known by the VLC receiver. Then, the unknown parameter vector in (5) becomes $\varphi=x$, and the Fisher information in (6) can be obtained, from (5), as

$$
\mathrm{J}(x)=\mathbb{E}\left\{\left(\frac{d \Lambda(x)}{d x}\right)^{2}\right\}=\left(\frac{R_{p} \alpha}{\sigma c}\right)^{2} E_{1}
$$

where

$$
E_{1} \triangleq \int_{0}^{T_{s}}\left(s^{\prime}(t)\right)^{2} d t
$$

with $s^{\prime}(t)$ denoting the derivative of $s(t)$ [24], [26]. Based on (7) and (9), the CRLB is computed as follows:

$$
\mathbb{E}\left\{(\hat{x}-x)^{2}\right\} \geq \frac{1}{E_{1}}\left(\frac{\sigma c}{R_{p} \alpha}\right)^{2} \triangleq \mathrm{CRLB}_{\mathrm{TOA}} .
$$

To provide an alternative expression for the CRLB in (11), $E_{1}$ in (10) is expressed, via Parseval's relation, as follows [24]:

$$
\begin{aligned}
E_{1} & =\int_{-\infty}^{\infty}|j 2 \pi f S(f)|^{2} d f=4 \pi^{2} \int_{-\infty}^{\infty} f^{2}|S(f)|^{2} d f \\
& =4 \pi^{2} \beta^{2} \int_{-\infty}^{\infty}|S(f)|^{2} d f=4 \pi^{2} E_{2} \beta^{2}
\end{aligned}
$$

where $S(f)$ denotes the Fourier transform of $s(t)$,

$$
E_{2} \triangleq \int_{-\infty}^{\infty}|S(f)|^{2} d f=\int_{0}^{T_{s}}(s(t))^{2} d t
$$

and $\beta$ is the effective bandwidth of $s(t)$ defined as

$$
\beta^{2}=\frac{1}{E_{2}} \int_{-\infty}^{\infty} f^{2}|S(f)|^{2} d f .
$$

From (12), (11) can be stated as

$$
\mathbb{E}\left\{(\hat{x}-x)^{2}\right\} \geq \frac{\sigma^{2} c^{2}}{4 \pi^{2} R_{p}^{2} \alpha^{2} E_{2} \beta^{2}} \triangleq \mathrm{CRLB}_{\mathrm{TOA}} .
$$

It is noted that the CRLB in (15) is equivalent to that in [6, eq. (5)] for $\sigma^{2}=N_{0} / 2$. Hence, the CRLB expression presented in [6] corresponds to a synchronous system in which the channel attenuation factor $\alpha$ is known by the VLC receiver but the relation of $\alpha$ to distance $x$ is unknown. Since only the time delay information is employed to estimate the distance, this scenario is referred to as TOA based distance estimation.

When the channel attenuation factor, $\alpha$, is unknown, the CRLB can be expressed for this scenario as in the following lemma. 
Lemma 1 [21]: When the channel attenuation factor $\alpha$ in (1) is unknown, the CRLB for TOA based distance estimation is given by

$$
\mathbb{E}\left\{(\hat{x}-x)^{2}\right\} \geq \frac{E_{2}}{E_{1} E_{2}-E_{3}^{2}}\left(\frac{\sigma c}{R_{p} \alpha}\right)^{2}
$$

where $E_{1}$ is as in (10), $E_{2}$ is given by (13), and

$$
E_{3} \triangleq \int_{0}^{T_{s}} s^{\prime}(t) s(t) d t=0.5\left(s^{2}\left(T_{s}\right)-s^{2}(0)\right)
$$

Proof: Please see Appendix A.

As expected, the CRLB in (16) is larger than or equal to the CRLB in (11) due to the presence of an additional unknown parameter. It is also observed that the CRLBs become equal when $E_{3}$ in (17) is equal to zero. Therefore, for $E_{3}=0$, the CRLB in [6] also corresponds to a synchronous system in which the channel attenuation factor $\alpha$ is unknown and the relation of $\alpha$ to distance $x$ is unavailable.

Secondly, the following assumptions are considered: $(i)$ the LED transmitter and the VLC receiver are synchronized (i.e., $\Delta=0$ in (2)) and (ii) the relation between channel attenuation factor $\alpha$ and distance $x$ is known, which is as stated in (4). The second assumption is practical for VLP systems since the channel attenuation factor can be specified accurately as a function of distance in LOS visible light channels.

In this scenario, the ML estimator can be obtained from (2) with $\Delta=0,(4)$, and (5) as follows ${ }^{4}$ :

$$
\begin{aligned}
\hat{x}_{\mathrm{ML}, \mathrm{hyb}}= & \underset{x}{\arg \max } x^{-m-3} \int_{T_{1}}^{T_{2}} r(t) s\left(t-\frac{x}{c}\right) d t \\
& -0.5 \gamma R_{p} x^{-2 m-6} E_{2} .
\end{aligned}
$$

Compared to the MLE in (8), the MLE in (18) also exploits the relation of the channel attenuation factor with the distance, as noted from the $x^{-m-3}$ and $x^{-2 m-6}$ terms.

Based on (2) with $\Delta=0$ and the relation in (4), the unknown parameter vector in (5) becomes $\varphi=x$. Then, from (4)-(6), the Fisher information can be calculated as

$$
\mathrm{J}(x)=\left(\frac{R_{p} \gamma}{\sigma x^{m+4}}\right)^{2} h_{1}(x)
$$

with

$$
h_{1}(x) \triangleq(m+3)^{2} E_{2}+2(m+3) \frac{x}{c} E_{3}+\frac{x^{2}}{c^{2}} E_{1}
$$

where $E_{1}, E_{2}$, and $E_{3}$ are given by (10), (13), and (17), respectively. From (7) and (19), the CRLB is computed as follows:

$$
\mathbb{E}\left\{(\hat{x}-x)^{2}\right\} \geq \frac{1}{h_{1}(x)}\left(\frac{\sigma x^{m+4}}{R_{p} \gamma}\right)^{2} \triangleq \mathrm{CRLB}_{\mathrm{hyb}} .
$$

The comparison between the CRLBs in (11) and (21) is provided in the following proposition:

\footnotetext{
${ }^{4}$ The meaning of subscript hyb (hybrid) will be clear towards the end of this section.
}

Proposition 1: The CRLB in (21) is smaller than that in (11) if and only if

$$
(m+3) E_{2}+\frac{2 x}{c} E_{3}>0 .
$$

Proof: First, the CRLB in (21) is expressed based on (4) as

$$
\mathbb{E}\left\{(\hat{x}-x)^{2}\right\} \geq \frac{x^{2}}{c^{2} h_{1}(x)}\left(\frac{\sigma c}{R_{p} \alpha}\right)^{2} .
$$

Then, the ratio of the CRLB in (11) to the CRLB in (23) is given by

$$
\begin{aligned}
\frac{c^{2} h_{1}(x)}{E_{1} x^{2}} & =\frac{c^{2}(m+3)^{2} E_{2}+2(m+3) x c E_{3}+x^{2} E_{1}}{E_{1} x^{2}} \\
& =1+\frac{c^{2}(m+3)^{2} E_{2}+2(m+3) x c E_{3}}{E_{1} x^{2}}
\end{aligned}
$$

where the relation in (20) is employed. Since $E_{1}, E_{2}, m$, $c$, and $x$ are positive by definition, the second term in (25) is positive if and only if the condition in (22) holds.

The condition in Proposition 1 commonly holds in practice since $x / c$ is very small (on the order of $10^{-8}$ for indoor scenarios) and/or $E_{3}$ is zero for many practical pulses [6]. Hence, the utilization of the relation in (4) is useful for improving the accuracy of distance estimation. From a practical point of view, this implies that instead of estimating (learning) the value of $\alpha$ first and then using that estimate in the TOA based distance estimation, a more efficient approach is to estimate the distance directly based on the model in (1) and (4) since the information in $\alpha$ related to distance $x$ is effectively utilized in that scenario. In other words, in the presence of the relation between the channel attenuation factor and the distance, information in both the channel attenuation factor and the time delay parameter are utilized for distance estimation. Hence, this scenario corresponds to hybrid TOA/RSS based distance estimation as the channel attenuation factor is related to RSS.

Remark 1: To illustrate the improvements that can be achieved by utilizing the relation between $\alpha$ and $x$, the relation in (25) can be considered for $E_{3}=0$, which becomes $1+c^{2}(m+3)^{2} E_{2} /\left(E_{1} x^{2}\right)$. From (12), this expression can be stated as $1+c^{2}(m+3)^{2} /\left(4 \pi^{2} \beta^{2} x^{2}\right)$. Hence, for typical system parameters, the CRLB for the TOA based distance estimation is significantly larger than the CRLB for the hybrid TOA/RSS based distance estimation for $\beta \ll c / x$, and they become comparable for high effective bandwidths (on the order of $100 \mathrm{MHz}$ or higher). As an example, for $x=10 \mathrm{~m}, m=1$, and $\beta=1 \mathrm{MHz}, 1+c^{2}(m+3)^{2} /\left(4 \pi^{2} \beta^{2} x^{2}\right)=365.76$, which means that the lower limit on the root MSEs (RMSEs) of unbiased estimators is 19.125 times smaller for the hybrid TOA/RSS based distance estimation than that for the TOA based distance estimation. On the other hand, when $\beta=100 \mathrm{MHz}$, $1+c^{2}(m+3)^{2} /\left(4 \pi^{2} \beta^{2} x^{2}\right)=1.0365$ is obtained, leading to comparable CRLBs.

\section{B. Case 2: Asynchronous System}

In this case, it is assumed the channel attenuation factor $\alpha$ and distance $x$ are related as in (4). However, the LED transmitter 
and the VLC receiver are not synchronized; that is, $\Delta$ in (2) is unknown. Hence, the delay parameter $\tau$ in (1) and (2) is modeled as an unknown parameter, and the vector of unknown parameters in (5) is specified by $\varphi=(x, \tau)$. Then, the ML estimator can be expressed based on (5) as follows:

$$
\begin{aligned}
\hat{x}_{\mathrm{ML}, \mathrm{RSS}}= & \underset{(x, \tau)}{\arg \max } x^{-m-3} \int_{T_{1}}^{T_{2}} r(t) s(t-\tau) d t \\
& -0.5 \gamma R_{p} x^{-2 m-6} E_{2}
\end{aligned}
$$

which can be re-stated as

$$
\hat{x}_{\mathrm{ML}, \mathrm{RSS}}=\underset{x}{\arg \max } x^{-m-3} \tilde{C}_{r s}-0.5 \gamma R_{p} x^{-2 m-6} E_{2}
$$

where

$$
\tilde{C}_{r s} \triangleq \max _{\tau} \int_{T_{1}}^{T_{2}} r(t) s(t-\tau) d t
$$

The solution of (27) can be obtained as

$$
\hat{x}_{\mathrm{ML}, \mathrm{RSS}}=\left(\frac{\gamma R_{p} E_{2}}{\tilde{C}_{r s}}\right)^{\frac{1}{m+3}}
$$

under the assumption that $\tilde{C}_{r s}$ is positive. It is noted that in the ML estimator in (26), the value of $\tau$ is estimated as the one that maximizes the correlation between the transmitted and received signals, as shown in (28). Then, that estimate is employed in the ML estimator, leading to the expression in (27).

Since the TOA parameter $\tau$ cannot be related to distance in this case due to asynchronism (see (2)), the distance estimation relies on the RSS information via (4) in this case, which is therefore referred to as RSS based distance estimation.

The CRLB for the RSS based distance estimation is given by the following lemma.

Lemma 2: For the signal model in (1), where the delay parameter is unknown and the channel attenuation factor is given by (4), the CRLB for distance estimation is expressed as

$$
\mathbb{E}\left\{(\hat{x}-x)^{2}\right\} \geq \frac{E_{1}}{E_{1} E_{2}-E_{3}^{2}}\left(\frac{\sigma x}{\alpha R_{p}(m+3)}\right)^{2} \triangleq \mathrm{CRLB}_{\mathrm{RSS}}
$$

where $E_{1}, E_{2}$, and $E_{3}$ are given by (10), (13), and (17), respectively.

Proof: Please see Appendix B.

It is noted that the CRLB expression in Lemma 2 covers that in [7] as a special case for $E_{3}=0$ (please see [7, eq. (15)]).

In the following proposition, the CRLB in Lemma 2 is compared to those corresponding to the TOA based and hybrid TOA/RSS based distance estimation.

Proposition 2: For $E_{3}=0$, the ratios of the CRLB in (30) to that in (21) and to that in (11) are expressed as

$$
\frac{\mathrm{CRLB}_{\mathrm{RSS}}}{\mathrm{CRLB}_{\mathrm{hyb}}}=1+\frac{4 \pi^{2} \beta^{2} x^{2}}{c^{2}(m+3)^{2}}=1+\frac{\mathrm{CRLB}_{\mathrm{RSS}}}{\mathrm{CRLB}_{\mathrm{TOA}}} .
$$

Proof: For $E_{3}=0$, the CRLB in (21) (equivalently, (23)) becomes

$$
\mathbb{E}\left\{(\hat{x}-x)^{2}\right\} \geq \frac{1}{(m+3)^{2} E_{2}+E_{1}(x / c)^{2}}\left(\frac{\sigma x}{R_{p} \alpha}\right)^{2} .
$$

Then, the ratio of the CRLB in (30) for $E_{3}=0$ to the CRLB in (32) is obtained as $1+E_{1} x^{2} /\left(E_{2} c^{2}(m+3)^{2}\right)$, which becomes equal to the central expression in (31) based on (12). In addition, the ratio of the CRLB in (30) for $E_{3}=0$ to the CRLB in (11) is given by $E_{1} x^{2} /\left(E_{2} c^{2}(m+3)^{2}\right)$, which is equal to $4 \pi^{2} \beta^{2} x^{2} /\left(c^{2}(m+3)^{2}\right)$ due to (12), leading to the second equality in (31).

Based on Proposition 2, the following conclusions are made:

- The CRLB for the RSS based distance estimation is very close to the CRLB for the hybrid TOA/RSS based distance estimation for practical indoor positioning systems when $\beta \ll c / x$. Since $x$ is less than $10 \mathrm{~m}$ in typical indoor scenarios, an effective bandwidth lower than about $1 \mathrm{MHz}$ results in approximately equal CRLBs (cf., Remark 1). In such a case, the distance related information gathered from the time delay parameter becomes negligible compared to the information gathered from the channel attenuation factor (equivalently, RSS).

- For $\beta \ll c / x$, the CRLB for the RSS based distance estimation is significantly lower than the CRLB for the TOA based distance estimation; that is, the RSS based distance estimation is much more accurate than the TOA based distance estimation.

- The TOA based distance estimation is more accurate than the RSS based distance estimation when $\beta>(m+$ $3) c /(2 \pi x)$. As an example, for $m=1$ and $x=5 \mathrm{~m}$, the effective bandwidth should satisfy $\beta>38.2 \mathrm{MHz}$ for the TOA based distance estimation to be more accurate.

- When $\beta$ is on the order of $(m+3) c /(2 \pi x)$, the hybrid TOA/RSS based distance estimation can provide nonnegligible improvements over both the TOA based and the RSS based distance estimation. When $\beta \gg c / x$, the CRLBs for the TOA based and hybrid TOA/RSS based distance estimation get very close.

Remark 2: Proposition 2 provides comparisons among different approaches based on the CRLBs (i.e., the distance estimation accuracy). On the other hand, with respect to implementation complexity, the RSS based distance estimation has an important practical advantage over the other approaches as it does not require synchronization between the clocks of the LED transmitter and the VLC receiver. Therefore, if the RSS based distance estimation can provide the required level of accuracy for an application, it can be the preferred approach. However, in some scenarios (e.g., for $\beta \gg c / x$ ), a synchronized system design may be required for achieving the desired accuracy level for distance estimation.

Remark 3: Based on the CRLB expressions obtained in this section, the effects of various parameters on the ranging accuracy can be analyzed. For example, the shape of the transmitted signal $s(t)$ can have different effects in the synchronous and asynchronous cases. For synchronous systems, the CRLB 
depends on the pulse shape via the $E_{1}$ parameter (equivalently, the effective bandwidth parameter $\beta$ in (12)). In particular, for signals with larger $E_{1}$ (equivalently, larger $\beta$ ), the TOA based CRLB in (15) and the hybrid TOA/RSS based CRLB in (20) and (21) get smaller; i.e., the accuracy improves ${ }^{5}$. On the other hand, for asynchronous systems, the RSS based CRLB in (30) does not depend on the pulse shape parameter, $E_{1}$, when $E_{3}=0$, which is commonly the case. As another important parameter, the height, $h$, can affect the accuracy of ranging systems. For instance, if the height parameter is increased while the irradiation angle $\phi$ and the incidence angle $\theta$ are unchanged, the distance between the LED transmitter and the VLC receiver increases. Then, it can be observed from (3) that the channel attenuation factor $\alpha$ reduces (i.e., the received power decreases) since the distance gets larger and the other parameters are fixed. Hence, based on (15), (21), and (30), all the CRLBs increase; that is, the accuracy degrades. On the other hand, if the height parameter is increased from $h$ to $\tilde{h}$ while the horizontal distance $D$ between the LED transmitter and the VLC receiver is kept the same, the accuracy can increase, decrease, or stay the same depending on the parameters $h, \tilde{h}, D$, and $m$, which can be analyzed based on (4), (15), (21), and (30).

\section{EFFECTS OF SAMPLING AND MODIFIED HYBRID ESTIMATOR}

It is noted from the MLEs in (8), (18), and (26) that the correlator outputs (i.e., the $\int_{T_{1}}^{T_{2}} r(t) s(t-x / c) d t$ and $\int_{T_{1}}^{T_{2}} r(t) s(t-$ $\tau) d t$ terms) should be evaluated for all possible distance (delay) values to obtain the ML distance estimates. However, in practical systems, it is costly and power consuming to obtain samples of correlator outputs (equivalently, matched filter outputs) at very high rates [27]. Therefore, it is important to investigate the effects of sampling rate limitations on the MSE performance of the MLEs. In this section, asymptotical analyses are performed (as the noise variance goes to zero) in order to quantify the effects of sampling.

Suppose that the correlator outputs are sampled at integer multiples of $T_{\mathrm{smp}}$ seconds, where $T_{\mathrm{smp}}$ denotes the sampling period. Also, the normalized autocorrelation function of signal $s(t)$ is defined as

$$
\rho(v) \triangleq \frac{1}{E_{2}} \int_{-\infty}^{\infty} s(t) s(t-v) d t .
$$

In the following lemma, the asymptotic performance of the TOA based and the RSS based ML distance estimation is specified in the presence of sampling rate limitations.

Lemma 3: Suppose that $\rho(v)>\rho(\varsigma), \quad \forall v \in[-0.5$ $\left.T_{\mathrm{smp}}, 0.5 T_{\mathrm{smp}}\right]$ and $\forall \varsigma \notin\left[-0.5 T_{\mathrm{smp}}, 0.5 T_{\mathrm{smp}}\right]$. Then, in the absence of noise (that is, for $\sigma=0$ ) and for a sampling period of $T_{\mathrm{smp}}$, the MSE of the TOA based MLE in (8) is

\footnotetext{
${ }^{5}$ For the hybrid TOA/RSS based scenario, if the information from the TOA parameter is negligible compared to that from the RSS parameter (i.e., if $\beta \ll$ $c / x$ ), then the hybrid TOA/RSS based CRLB does not change significantly with the pulse shape $\left(E_{1}\right.$ or $\left.\beta\right)$.
}

given by

$$
\mathrm{MSE}_{\mathrm{TOA}}=\left(x-c T_{\mathrm{smp}} \text { round }\left(\frac{x}{c T_{\mathrm{smp}}}\right)\right)^{2}
$$

and the MSE of the RSS based MLE in (29) is expressed as

$$
\operatorname{MSE}_{\mathrm{RSS}}=x^{2}\left(1-\left(\rho\left(\tau-T_{\mathrm{smp}} \operatorname{round}\left(\frac{\tau}{T_{\mathrm{smp}}}\right)\right)\right)^{\frac{-1}{m+3}}\right)^{2}
$$

where $x$ is the distance between the LED transmitter and the VLC receiver, $\tau=x / c+\Delta$ as stated in (2), $\rho(\cdot)$ is as defined in (33), and round $(y)$ represents the closest integer to $y$.

Proof: The expression in (34) simply follows from (8) based on (1) without noise. In particular, for a sampling period of $T_{\mathrm{smp}}$ and for $\sigma=0$, (8) becomes

$$
\hat{x}_{\mathrm{ML}, \mathrm{TOA}}=\underset{i c T_{\mathrm{smp}}}{\arg \max } \alpha R_{p} E_{2} \rho\left(\frac{x-i c T_{\mathrm{smp}}}{c}\right)
$$

where $i$ is an integer, $x$ denotes the true distance, and $\rho(\cdot)$ is as in (33). Under the assumption in the lemma, the autocorrelation term in (36) is maximized for $i=\operatorname{round}\left(x /\left(c T_{\mathrm{smp}}\right)\right)$. Hence, the ML estimate becomes $\hat{x}_{\mathrm{ML}, \mathrm{TOA}}=$ $c T_{\mathrm{smp}} \operatorname{round}\left(x /\left(c T_{\mathrm{smp}}\right)\right)$ and the (mean) squared error is obtained as in (34).

For the RSS based ML estimator in (29), $\tilde{C}_{r s}$ in (28) can be calculated, for a sampling period of $T_{\mathrm{smp}}$ and for $\sigma=0$, as

$$
\begin{aligned}
\tilde{C}_{r s} & =\max _{i T_{\mathrm{smp}}} \alpha R_{p} E_{2} \rho\left(\tau-i T_{\mathrm{smp}}\right) \\
& =\alpha R_{p} E_{2} \rho\left(\tau-T_{\mathrm{smp}} \operatorname{round}\left(\tau / T_{\mathrm{smp}}\right)\right)
\end{aligned}
$$

where $\tau=x / c+\Delta$ denotes the time delay as stated in (2), and the assumption in the lemma is employed to obtain the final expression. Then, the RSS based ML estimator in (29) becomes

$$
\hat{x}_{\mathrm{ML}, \mathrm{RSS}}=\left(\frac{\gamma R_{p} E_{2}}{\alpha R_{p} E_{2} \rho\left(\tau-T_{\mathrm{smp}} \operatorname{round}\left(\tau / T_{\mathrm{smp}}\right)\right)}\right)^{\frac{1}{m+3}}
$$

which can be expressed via (4) as

$$
\hat{x}_{\mathrm{ML}, \mathrm{RSS}}=\frac{x}{\left(\rho\left(\tau-T_{\mathrm{smp}} \operatorname{round}\left(\tau / T_{\mathrm{smp}}\right)\right)\right)^{\frac{1}{m+3}}} .
$$

From (40), the (mean) squared error can be obtained as in (35).

The assumption in Lemma 3 commonly holds in practice for a sufficiently small $T_{\mathrm{smp}}$. For example, $\rho(v)$ in (33) corresponding to $s(t)$ in (50) is presented in Fig. 1 for $T_{s}=0.1 \mathrm{~ms}$, $f_{c}=100 \mathrm{kHz}$, and $A=0.1$. It is observed that the assumption in Lemma 3 holds for $T_{\mathrm{smp}}<1 \mu \mathrm{s}$; that is, when the sampling rate is higher than $1 \mathrm{MHz}$. It should be noted that high sampling rates are already required for accurate distance estimation; hence, the assumption is Lemma 3 is realistic for most practical applications.

From Lemma 3, it is deduced that the TOA based MLE is directly affected from the mismatches between the sampling time instant and the true delay of the incoming signal whereas the effects on the RSS based MLE is through the sensitivity of 


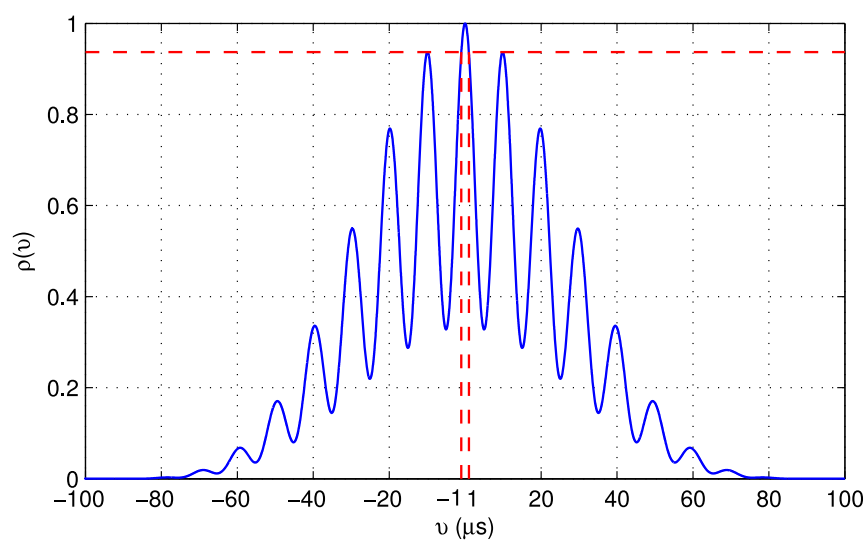

Fig. 1. Normalized autocorrelation function in (33) for $s(t)$ in (50) with $T_{s}=0.1 \mathrm{~ms}, f_{c}=100 \mathrm{kHz}$, and $A=0.1$.

the normalized autocorrelation function, $\rho(v)$, to timing mismatches. For example, if $\rho(v)$ does not change significantly for $v \in\left[-0.5 T_{\mathrm{smp}}, 0.5 T_{\mathrm{smp}}\right]$, then effects of the sampling rate can become negligible for the RSS based MLE. Also, it is noted from (34) and (35) that, depending on the value of distance $x$ and the time delay, the maximum squared error due to sampling is equal to $\left(0.5 c T_{\mathrm{smp}}\right)^{2}$ for the TOA based MLE and it is given by $x^{2}\left(1-\left(\rho\left(0.5 T_{\mathrm{smp}}\right)\right)^{-1 /(m+3)}\right)^{2}$ for the RSS based MLE.

For the asymptotic performance of the hybrid TOA/RSS based MLE, the following lemma is presented.

Lemma 4: Define the following function

$$
g_{x}(u) \triangleq(u x)^{-m-3} \rho\left(\frac{x-u}{c}\right)-0.5 u^{-2 m-6}
$$

where $x$ denotes the distance between the LED transmitter and the VLC receiver and $\rho$ is as in (33). Assume that $g_{x}(u)>g_{x}(v), \forall u \in\left[x, x+c T_{\mathrm{smp}}\right]$ and $\forall v>x+c T_{\mathrm{smp}}$, and that $g_{x}(u)>g_{x}(v), \forall u \in\left[x-c T_{\mathrm{smp}}, x\right]$ and $\forall v<x-c T_{\mathrm{smp}}$. In addition, define $i_{1}$ and $i_{2}$ as

$$
i_{1} \triangleq\left\lfloor\frac{x}{c T_{\mathrm{smp}}}\right\rfloor, i_{2} \triangleq\left\lceil\frac{x}{c T_{\mathrm{smp}}}\right\rceil
$$

where $\lfloor y\rfloor$ denotes the largest integer smaller than or equal to $y$ and $\lceil y\rceil$ represents the smallest integer larger than or equal to $y$. Then, the MSE of the hybrid TOA/RSS based MLE in (18) is expressed as

$$
\mathrm{MSE}_{\mathrm{hyb}}=\left(x-\hat{i} c T_{\mathrm{smp}}\right)^{2}
$$

where

$$
\hat{i}=\underset{i \in\left\{i_{1}, i_{2}\right\}}{\arg \max } g_{x}\left(i c T_{\mathrm{smp}}\right) .
$$

Proof: In the absence of noise, $r(t)$ in (1) becomes $r(t)=$ $\alpha R_{p} s(t-x / c)$ for a synchronized system, where $x$ is the distance between the LED transmitter and the VLC receiver. Replacing the dummy variable $x$ in (18) with $u$, and then inserting $r(t)=\alpha R_{p} s(t-x / c)$, the objective function for the hybrid TOA/RSS based MLE in (18) can be expressed as

$$
u^{-m-3} \alpha R_{p} E_{2} \rho\left(\frac{x-u}{c}\right)-0.5 \gamma R_{p} u^{-2 m-6} E_{2}
$$

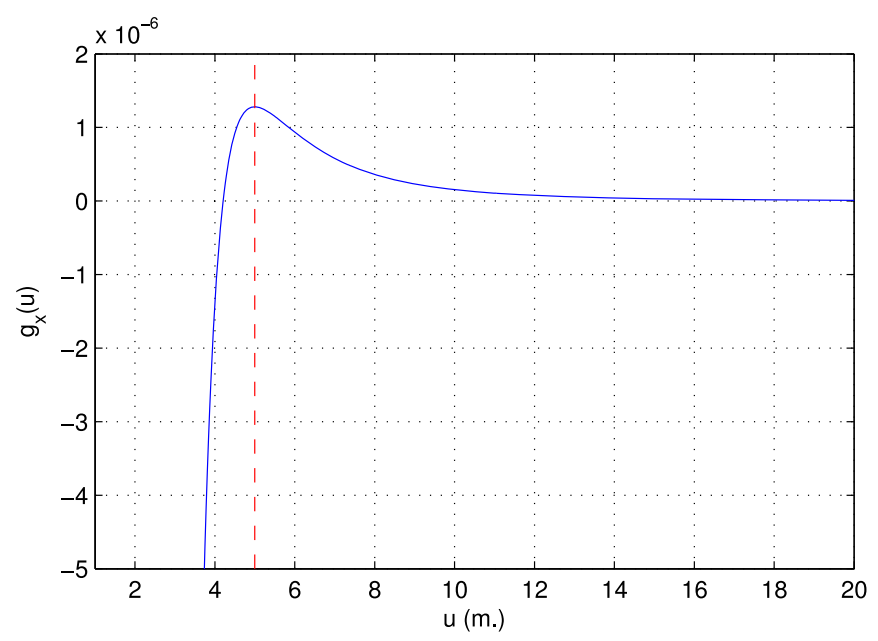

Fig. 2. Function $g_{x}(u)$ in (41) for $s(t)$ in (50), where $x=5 \mathrm{~m}, T_{s}=0.1 \mathrm{~ms}$, $f_{c}=100 \mathrm{kHz}$, and $A=0.1$.

where $\rho$ is given by (33). Based on (4), (45) can be expressed as

$$
\begin{aligned}
& \gamma R_{p} E_{2}\left(u^{-m-3} x^{-m-3} \rho\left(\frac{x-u}{c}\right)-0.5 u^{-2 m-6}\right) \\
& \triangleq \gamma R_{p} E_{2} g_{x}(u)
\end{aligned}
$$

where the equality follows from (41). For a sampling period of $T_{\mathrm{smp}}$, the hybrid TOA/RSS based ML estimator in (18) can be stated based on (46) as

$$
\hat{x}_{\mathrm{ML}, \mathrm{hyb}}=\underset{i c T_{\mathrm{smp}}}{\arg \max } \gamma R_{p} E_{2} g_{x}\left(i c T_{\mathrm{smp}}\right) .
$$

Under the assumptions in the lemma about $g_{x}(\cdot)$, the MLE in (47) becomes equal to either $i_{1} c T_{\mathrm{smp}}$ or $i_{2} c T_{\mathrm{smp}}$, where $i_{1}$ and $i_{2}$ are as in (42). If $g_{x}\left(i_{1} c T_{\mathrm{smp}}\right)>g_{x}\left(i_{2} c T_{\mathrm{smp}}\right)$, then $\hat{x}_{\mathrm{ML}, \text { hyb }}=$ $i_{1} c T_{\mathrm{smp}}$; otherwise, $\hat{x}_{\mathrm{ML}, \mathrm{hyb}}=i_{2} c T_{\mathrm{smp}}$. Hence, the (mean) squared error can expressed as in (43) and (44).

It can be shown that $g_{x}(u)$ in (41) achieves the maximum value at $u=x$. Hence, the assumption in Lemma 4 is valid for practical scenarios for a sufficiently small $T_{\mathrm{smp}}$ and as long as the normalized autocorrelation function, $\rho((x-u) / c)$, does not change rapidly compared to $u^{-m-3}$. In Fig. $2, g_{x}(u)$ is presented for $s(t)$ in (50), where $x=5 \mathrm{~m}, T_{s}=0.1 \mathrm{~ms}, f_{c}=100 \mathrm{kHz}$, and $A=0.1$. It is observed that the assumption in Lemma 4 holds for all values of $T_{\mathrm{smp}}$ in this case.

Lemma 4 indicates that, similar to the TOA based MLE, the hybrid TOA/RSS based MLE is directly affected from the mismatches between the sampling time instant and the true delay of the incoming signal, and it is subject to a maximum squared error of $\left(0.5 c T_{\mathrm{smp}}\right)^{2}$ due to sampling.

For high distance estimation accuracy, the maximum absolute error of $0.5 c T_{\mathrm{smp}}$ can be quite undesirable. For example, for a sampling period of $T_{\mathrm{smp}}=1 \mathrm{~ns}$, the absolute error induced by sampling can be as high as $15 \mathrm{~cm}$. Hence, the accuracy limits promised by the CRLBs may not be achievable. To alleviate this problem, a modified version of the hybrid TOA/RSS based ML estimator is proposed in this section. The modified hybrid TOA/RSS based estimator is implemented in two steps: 
(i) Obtain the hybrid TOA/RSS based ML estimate $\hat{x}_{\mathrm{ML} \text {,hyb }}$ from (18).

(ii) Calculate the final distance estimate as

$$
\hat{x}_{\text {modi-hyb }}=\left(\frac{\gamma R_{p} E_{2}}{\int_{T_{1}}^{T_{2}} r(t) s\left(t-\hat{x}_{\mathrm{ML}, \mathrm{hyb}} / c\right) d t}\right)^{\frac{1}{m+3}} .
$$

The main intuition behind the modified hybrid TOA/RSS based estimator is as follows: When the estimate $\hat{x}_{\mathrm{ML}, \mathrm{hyb}}$ in (18) is obtained in the presence of sampling errors, the correlator term $\int_{T_{1}}^{T_{2}} r(t) s(t-x / c) d t$ in (18) can be evaluated for $x=\hat{x}_{\mathrm{ML} \text {,hyb }}$ and then the distance estimate can be obtained with higher resolution by calculating the maximizer of $x^{-m-3} \int_{T_{1}}^{T_{2}} r(t) s\left(t-\hat{x}_{\mathrm{ML}, \mathrm{hyb}} / c\right) d t-0.5 \gamma R_{p} x^{-2 m-6} E_{2}$ as in (48) (similar to (29)).

Under the conditions in Lemma 4, the MSE of the modified hybrid TOA/RSS based estimator in (48) can be expressed in the absence of noise and for a sampling period of $T_{\mathrm{smp}}$ as ${ }^{6}$

$$
\operatorname{MSE}_{\text {mod }}=x^{2}\left(1-\left(\rho\left(\tau-\hat{i} T_{\mathrm{smp}}\right)\right)^{\frac{-1}{m+3}}\right)^{2}
$$

where $\hat{i}$ is as in (44). It is noted from (49) that, similar to the RSS based MLE, the modified hybrid TOA/RSS based estimator is affected from the sampling induced errors through the normalized autocorrelation function, and it is subject to a maximum squared error of $x^{2}\left(1-\left(\rho\left(0.5 T_{\mathrm{smp}}\right)\right)^{-1 /(m+3)}\right)^{2}$ due to sampling. Hence, when the normalized autocorrelation function is not very sensitive to timing mismatches, the modified hybrid TOA/RSS based estimator can have robustness against the effects of sampling.

\section{NumericAl RESUltS}

In this section, numerical examples are presented to investigate the theoretical limits and the MLEs for different approaches. A system model similar to that in [6] is considered. Namely, the Lambertian order is taken as $m=1, h$ in (4) is set to $2.5 \mathrm{~m}$, and the responsivity of the photo detector is given by $R_{p}=0.4 \mathrm{~mA} / \mathrm{mW}$. In addition, the area $A_{R}$ of the photo detector at the VLC receiver is equal to $1 \mathrm{~cm}^{2}$, and the spectral density level of the noise is set to $\sigma^{2}=1.336 \times 10^{-22} \mathrm{~W} / \mathrm{Hz}$ based on the employed parameters in $[6]^{7}$. Signal $s(t)$ in (1) is modeled as follows [6]:

$$
s(t)=A\left(1-\cos \left(\frac{2 \pi t}{T_{s}}\right)\right)\left(1+\cos \left(2 \pi f_{c} t\right)\right)
$$

for $t \in\left[0, T_{s}\right]$, where $f_{c}$ is the center frequency, and $A$ corresponds to the average emitted optical power (i.e., source optical

\footnotetext{
${ }^{6}$ The derivation is not presented as it is similar to that in Lemma 3.

${ }^{7}$ From [6, eq. (18)], $\sigma^{2}=q R_{p} p_{n} A_{R} \Delta \lambda$, where $q$ denotes the charge on an electron, $p_{n}=5.8 \times 10^{-6} \mathrm{~W} / \mathrm{cm}^{2} \cdot \mathrm{nm}$ is the background spectral irradiance, and $\Delta \lambda=360 \mathrm{~nm}$ is the bandwidth of the optical filter in front of the photodiode. (It should be noted that the results in the previous sections are valid for a generic zero-mean Gaussian noise component, which can consist of any types of noise such as shot noise and thermal noise.)
}

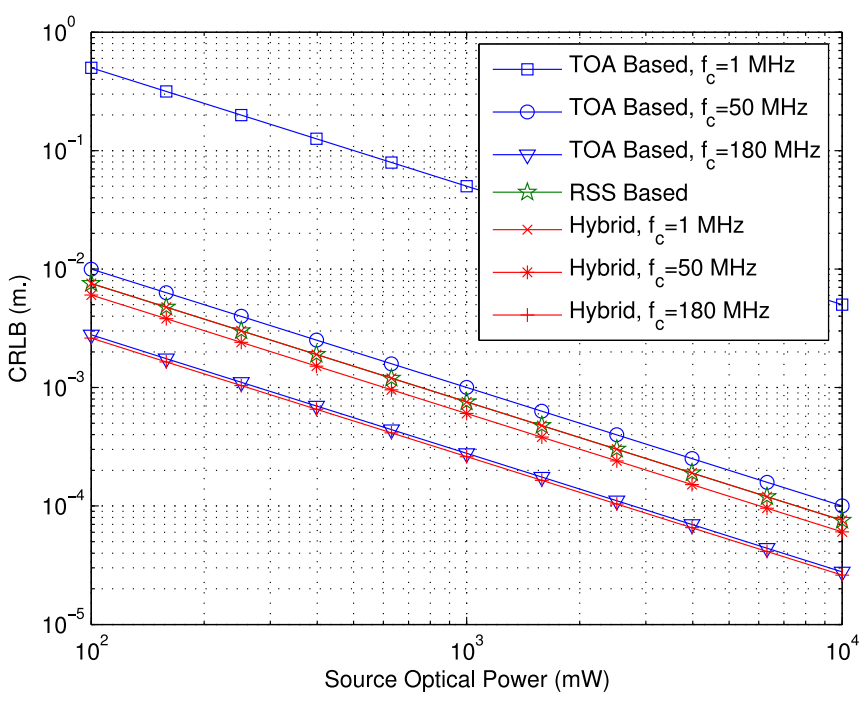

Fig. 3. CRLB versus source optical power for TOA based, hybrid TOA/RSS based, and RSS based approaches, where $x=5 \mathrm{~m}$ and $T_{s}=0.01 \mathrm{~s}$.

power). For $f_{c} \gg 1 / T_{s}$, it can be shown that the electrical energy of $s(t)$ defined in (13) and the effective bandwidth of $s(t)$ specified by (14) can be approximated as $E_{2}=9 A^{2} T_{s} / 4$ and $\beta=f_{c} / \sqrt{3}$, respectively [6]. In addition, parameter $E_{3}$ in (17) is obtained as $E_{3}=0$ for the signal in (50).

First, the CRLBs are calculated for $T_{s}=0.01 \mathrm{~s}$. when the distance between the LED transmitter and the VLC receiver is given by $x=5 \mathrm{~m}$. In Fig. 3, the CRLBs are plotted versus the source optical power $A$ for the TOA based, hybrid TOA/RSS based, and RSS based approaches considering different center frequencies. As expected, the hybrid TOA/RSS approach achieves the minimum CRLB in all cases since it utilizes information from both the time delay and channel attenuation factor. It is also noted that the performance of the RSS based distance estimation does not depend on the center frequency. This is due to the fact that RSS information is related to the energy of the signal but does not change with the other signal characteristics, which can be observed from (30) in Lemma 2 for $E_{3}=0$; that is, $\mathrm{CRLB}_{\mathrm{RSS}}=\sigma^{2} x^{2} /\left(E_{2} \alpha^{2} R_{p}^{2}(m+3)^{2}\right)$. Another observation from Fig. 3 is that the TOA based distance estimation has significantly higher CRLBs than the other approaches for relatively low center frequencies, for which the RSS based and hybrid TOA/RSS based approaches achieve almost the same accuracy (as the distance related information obtained from the TOA parameter becomes negligible). On the other hand, the TOA based distance estimation achieves lower CRLBs than the RSS based approach for high center frequencies; e.g., $f_{c}=180 \mathrm{MHz}$ [28], [29]. In that case, the information obtained from the TOA parameter becomes more significant than that extracted from the RSS parameter (channel attenuation factor), and the TOA based and hybrid TOA/RSS based approaches have almost the same performance. All these observations are in accordance with the relation in Proposition 2.

In order to provide further insights, the theoretical limits are plotted versus $f_{c}$ in Fig. 4 for the TOA based, hybrid TOA/RSS based, and RSS based approaches, where $x=5 \mathrm{~m}$. and $A=0.1$. 


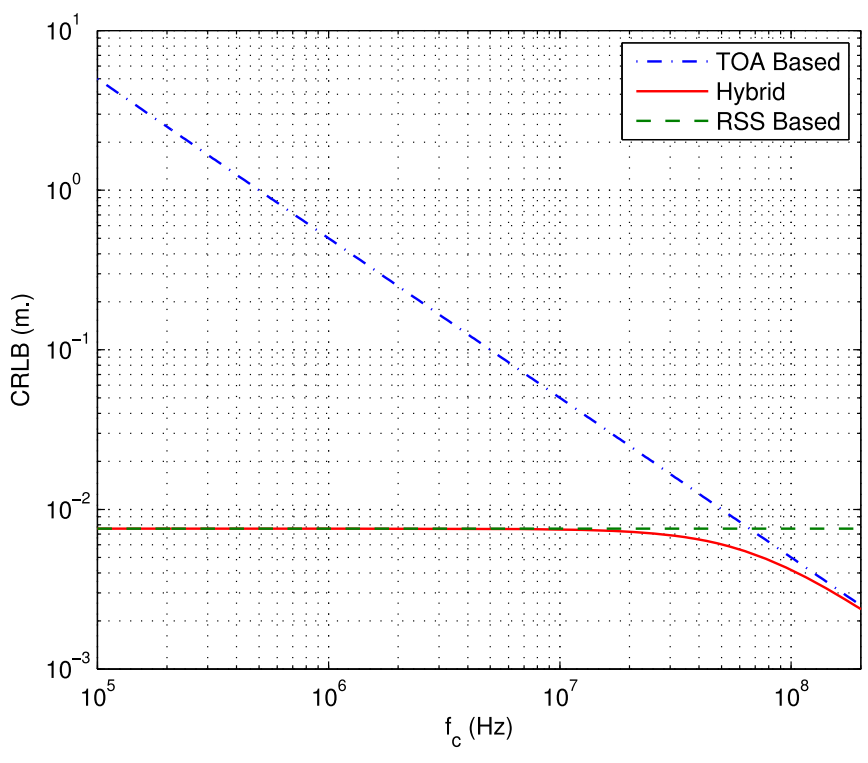

Fig. 4. CRLB versus $f_{c}$ for TOA based, hybrid TOA/RSS based, and RSS based approaches, where $x=5 \mathrm{~m}$ and $A=0.1$.

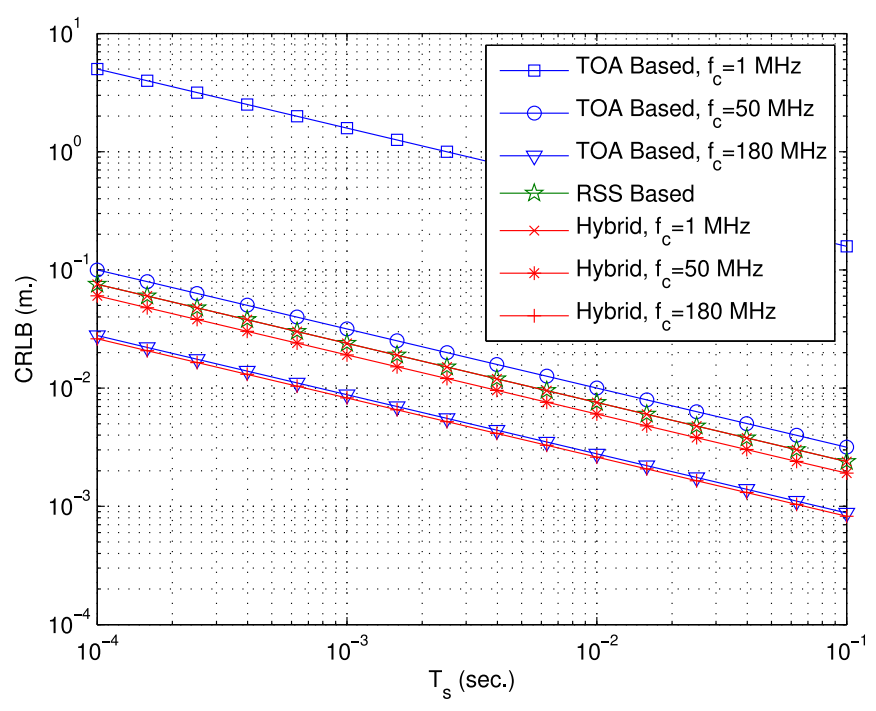

Fig. 5. CRLB versus $T_{s}$ for TOA based, hybrid TOA/RSS based, and RSS based approaches, where $x=5 \mathrm{~m}$ and $A=0.1$.

It is observed that the accuracy of the TOA based distance estimation improves with $f_{c}$ since $E_{1}$ in (12) increases with $f_{c}$. Also, there exists a critical frequency, which is equal to 66.16 MHz in this scenario, after (before) which the TOA based distance estimation achieves a lower (higher) CRLB than the RSS based approach. It is also noted that the hybrid TOA/RSS based approach provides nonnegligible improvements over both the TOA based and RSS based approaches around that critical frequency.

Next, the CRLBs are plotted versus the signal duration $T_{s}$ in Fig. 5 for the TOA based, hybrid TOA/RSS based, and RSS based approaches, where $x=5 \mathrm{~m}$. and $A=0.1$. As the signal energy increases with $T_{s}$ (note that $E_{2}=9 A^{2} T_{s} / 4$ ), the performance of distance estimation improves with $T_{s}$, as expected. As in Fig. 3, it is observed that the TOA based distance

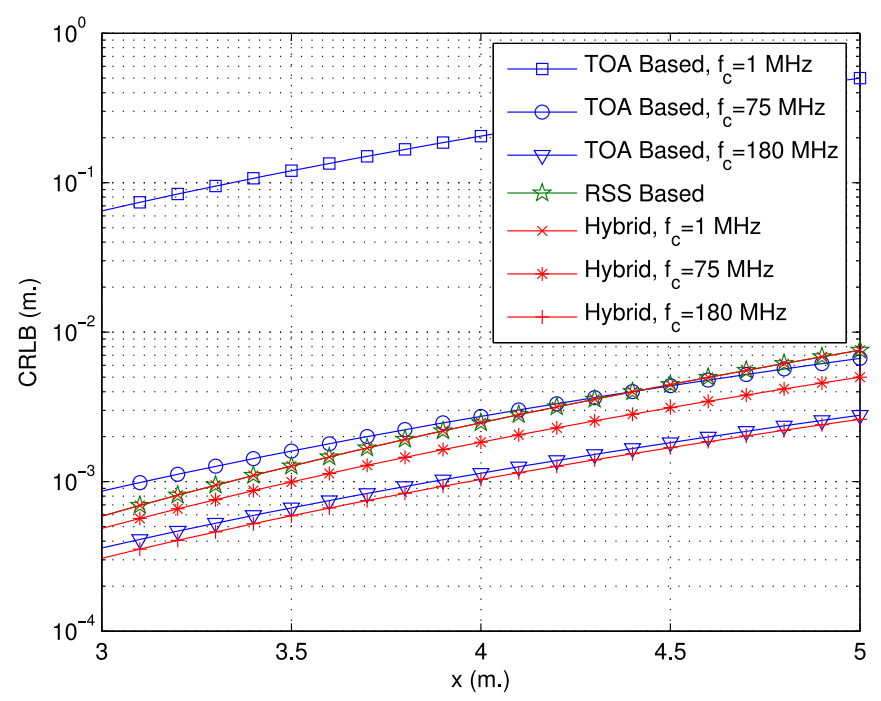

Fig. 6. CRLB versus distance $x$ for TOA based, hybrid TOA/RSS based, and RSS based approaches, where $T_{s}=0.01 \mathrm{~s}$ and $A=0.1$.

estimation achieves lower (higher) CRLBs than RSS based distance estimation for higher (lower) center frequencies. It is also noted that for the RSS based distance estimation to achieve a CRLB of $1 \mathrm{~cm}$, the signal duration should be around $6 \mathrm{~ms}$. On the other hand, shorter signal durations can be employed by the TOA based and hybrid TOA/RSS based approaches for high center frequencies (e.g., $T_{s} \approx 0.6-0.7 \mathrm{~ms}$. for $f_{c}=180 \mathrm{MHz}$.).

In Fig. 6, the CRLBs are plotted versus the distance $x$ between the LED transmitter and the VLC receiver for $f_{c}=1 \mathrm{MHz}$, $f_{c}=75 \mathrm{MHz}$, and $f_{c}=180 \mathrm{MHz}$, where $T_{s}=0.01 \mathrm{~s}$ and $A=$ 0.1 . It is intuitive that the estimation accuracy degrades (i.e., the CRLBs increase) as the distance gets larger. This intuitive observation is also verified by the expressions in (11), (21), and (30) via the relations in (4) and (20). Also, it is noted from Fig. 6 that in some cases (e.g., for $f_{c}=75 \mathrm{MHz}$ ) the RSS based distance estimation can have lower CRLBs than the TOA based approach up to a certain distance and then it results in higher CRLBs after that distance. This is due to fact that the CRLB (in meters) increases with $x^{m+4}$ for the RSS based approach whereas it increases with $x^{m+3}$ for the TOA based approach, as can be deduced from (4), (11), and (30).

It should be emphasized that although the comparisons in Figs. 3-6 are based on the CRLBs (i.e., the distance estimation accuracy), implementation complexity should also be considered for practical applications. As stated in Remark 2, the RSS based distance estimation has an important practical advantage over the other approaches since it does not require synchronization between the clocks of the LED transmitter and the VLC receiver. Hence, if the RSS based distance estimation can provide the required level of accuracy for an application, it can be the preferred approach. Otherwise, a synchronized system design may be required for achieving the desired accuracy level for distance estimation.

Finally, the MLEs in Sections III and IV are implemented and compared for a scenario with $x=5 \mathrm{~m} ., T_{s}=0.1 \mathrm{~ms}, f_{c}=$ $1 \mathrm{MHz}, \Delta=0$ (see (2)), and $T_{\mathrm{smp}}=1 \mathrm{~ns}$. In Fig. 7, the RMSEs 


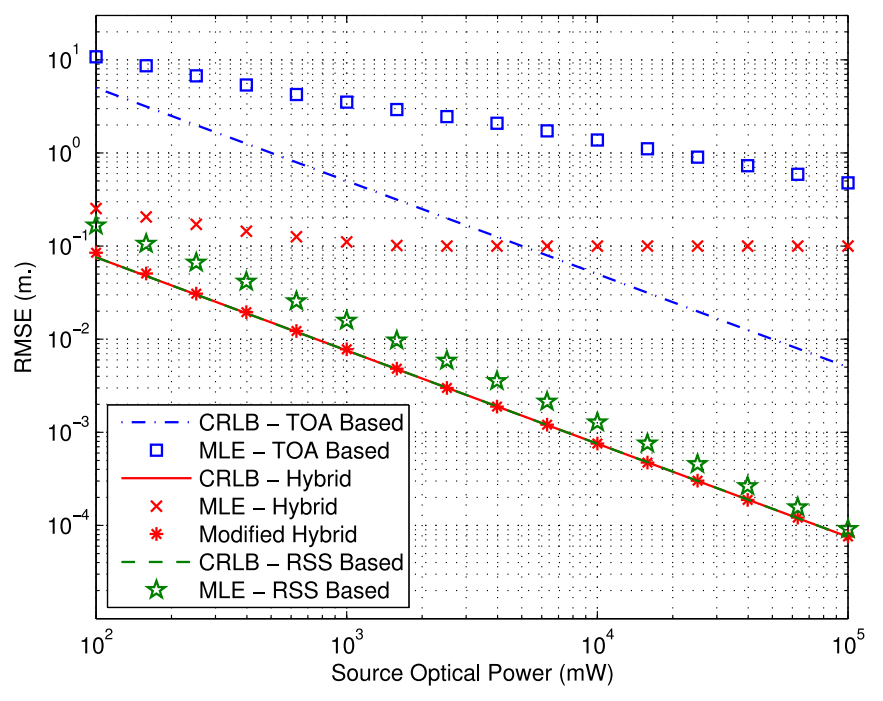

Fig. 7. RMSEs of the MLEs and the CRLBs for different approaches, where $x=5 \mathrm{~m}, T_{s}=0.1 \mathrm{~ms}, f_{c}=1 \mathrm{MHz}$, and $T_{\mathrm{smp}}=1 \mathrm{~ns}$.

of the TOA based MLE in (8), the hybrid TOA/RSS based MLE in (18), the RSS based MLE in (29), and the modified hybrid TOA/RSS based estimator in (48) are illustrated along with the CRLBs ${ }^{8}$. As expected from the analysis in Section IV, the TOA based MLE and the hybrid TOA/RSS based MLE are directly affected by the sampling rate limitation and their RMSEs converge towards $0.1 \mathrm{~m}$. in accordance with (34) and (43). On the other hand, the asymptotic RMSEs of the RSS based MLE and the modified hybrid TOA/RSS based estimator are calculated from (35) and (49) as $9.14 \times 10^{-7} \mathrm{~m}$., which is outside the practical accuracy range. Hence, the sampling rate limitation does not have any significant effects on these estimators in this scenario. It is also noted that the modified hybrid TOA/RSS based estimator converges to the CRLB faster than the RSS based MLE, and achieves the best performance for all power levels of interest. In addition, the hybrid TOA/RSS based MLE has lower CRLBs than the TOA based MLE since it utilizes both the time delay and RSS information. In Fig. 8, the RMSEs of the MLEs are plotted versus $T_{\mathrm{smp}}$ in the absence of noise to investigate the effects of the sampling period, where $x=5 \mathrm{~m}, T_{s}=0.1 \mathrm{~ms}, f_{c}=1 \mathrm{MHz}$, and $\Delta=0$. In the figure, the sampling period $T_{\mathrm{smp}}$ is incremented with a step size of $10^{-12} \mathrm{~s}$. It is observed that the RMSEs of the MLEs fluctuate as $T_{\mathrm{smp}}$ changes, which is due to the fact that the RMSE converges towards zero as the distance, $x$, gets close to an integer multiple of $c T_{\mathrm{smp}}$ (where $c$ is the speed of light). This observation can also be verified based on (34), (35), (43), and (49). In addition, Fig. 8 indicates that the local averages of the RMSEs reduce in general as the sampling rate increases (i.e., as $T_{\mathrm{smp}}$ decreases). Furthermore, the asymptotic RMSEs of the modified hybrid TOA/RSS based MLE and the RSS based MLE are observed to be outside the practical accuracy limits whereas those of the TOA based MLE and the hybrid TOA/RSS based MLE are in

\footnotetext{
${ }^{8}$ The search space for possible distance values is set to $[0,100] \mathrm{m}$. for all the estimators. Therefore, the MLEs in Fig. 7 can also be considered as maximum $a$ posteriori probability estimators [24] for a uniform prior distribution of $x$ over $[0,100] \mathrm{m}$.
}

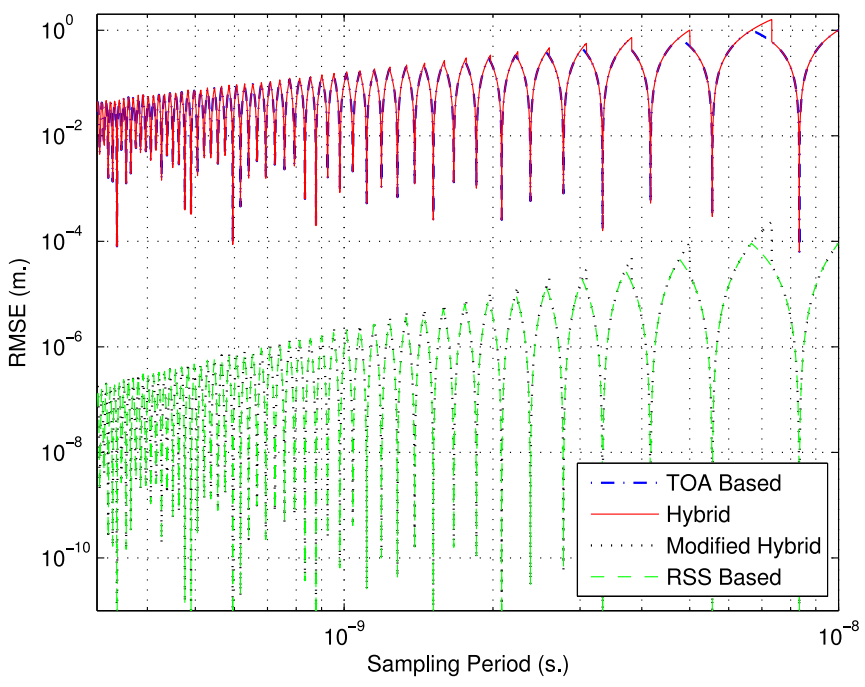

Fig. 8. RMSEs of the MLEs for different approaches in the absence of noise, where $x=5 \mathrm{~m}, T_{s}=0.1 \mathrm{~ms}$, and $f_{c}=1 \mathrm{MHz}$.

the range of practical accuracy limits. Hence, the sampling rate limitation can be crucial for the TOA based MLE and the hybrid TOA/RSS based MLE.

\section{RELATION TO POSITION ESTIMATION}

Wireless position estimation is commonly performed in two steps, where position related parameters such as distances or angles are estimated in the first step and the position is estimated based on those estimated parameters in the second step [27]. Therefore, distance estimation investigated in this study can be considered as the first step in a wireless localization system. As the accuracy of distance estimation improves, position estimation also gets more accurate in general. To present a formal relation between position estimation and distance estimation accuracy, let $\boldsymbol{l}_{r}=\left[l_{r, 1} l_{r, 2} l_{r, 3}\right]$ denote the location of the VLC receiver, and $\boldsymbol{l}_{t_{1}}, \ldots, \boldsymbol{l}_{t_{N}}$, with $\boldsymbol{l}_{t_{i}}=\left[l_{t_{i}, 1} l_{t_{i}, 2} l_{t_{i}, 3}\right]$, represent the known locations of the LED transmitters, which are utilized for the localization of the VLC receiver. For sufficiently high signal-to-noise ratios (SNRs) (which is commonly the case in LOS visible light channels), the ML estimate for the distance between the VLC receiver and the $i$ th LED transmitter can be stated as

$$
\hat{x}_{i}=x_{i}+\varsigma_{i}
$$

for $i=1, \ldots, N$, where the noise components $\varsigma_{1}, \ldots, \varsigma_{N}$ are independent, $x_{i}=\left\|\boldsymbol{l}_{r}-\boldsymbol{l}_{t_{i}}\right\|_{2}$, and $\varsigma_{i}$ is modeled as a zeromean Gaussian random variable with a variance that is equal to $\mathrm{CRLB}_{i}$, i.e., the CRLB for estimating $x_{i}$ based on the received signal coming from the $i$ th LED transmitter [24], [30]. In other words, at high SNRs, the ML estimate for the distance is modeled by a Gaussian random variable with a mean that is equal to the true distance and a variance that is equal to the CRLB [24], [30]. It is noted that the results in Section III specify $\mathrm{CRLB}_{i}$ for various estimation approaches (TOA based, RSS based, and TOA/RSS based). 
The CRLB for estimating the position $\boldsymbol{l}_{r}$ of the VLC receiver based on $\hat{x}_{1}, \ldots, \hat{x}_{N}$ can be expressed as [24]

$$
\mathrm{E}\left\{\left\|\hat{\boldsymbol{l}}_{r}-\boldsymbol{l}_{r}\right\|^{2}\right\} \geq \operatorname{trace}\left\{\mathbf{J}\left(\boldsymbol{l}_{r}\right)^{-1}\right\}
$$

where $\mathbf{J}\left(\boldsymbol{l}_{r}\right)$ denotes the FIM related to $\boldsymbol{l}_{r}$ (cf., (6)). Since the height of the VLC receiver is assumed to be known (cf., Section II), the aim is to estimate the first two elements of $\boldsymbol{l}_{r}$; that is, $l_{r, 1}$ and $l_{r, 2}$. Hence, based on (6), the FIM can be specified for the model in (51) as follows:

$$
\begin{aligned}
& {\left[\mathbf{J}\left(\boldsymbol{l}_{r}\right)\right]_{11}=\sum_{i=1}^{N} \frac{\left(l_{t_{i}, 1}-l_{r, 1}\right)^{2}}{\mathrm{CRLB}_{i} x_{i}^{2}},\left[\mathbf{J}\left(\boldsymbol{l}_{r}\right)\right]_{22}=\sum_{i=1}^{N} \frac{\left(l_{t_{i}, 2}-l_{r, 2}\right)^{2}}{\mathrm{CRLB}_{i} x_{i}^{2}},} \\
& {\left[\mathbf{J}\left(\boldsymbol{l}_{r}\right)\right]_{12}=\left[\mathbf{J}\left(\boldsymbol{l}_{r}\right)\right]_{21}=\sum_{i=1}^{N} \frac{\left(l_{t_{i}, 1}-l_{r, 1}\right)\left(l_{t_{i}, 2}-l_{r, 2}\right)}{\mathrm{CRLB}_{i} x_{i}^{2}} .}
\end{aligned}
$$

Then, the CRLB in (52) is calculated as

$$
\begin{aligned}
& \mathrm{E}\left\{\left\|\hat{\boldsymbol{l}}_{r}-\boldsymbol{l}_{r}\right\|^{2}\right\} \geq\left(\sum_{i=1}^{N} \frac{1}{\mathrm{CRLB}_{i}}\right)\left(\sum_{i=1}^{N} \frac{\left(l_{t_{i}, 1}-l_{r, 1}\right)^{2}}{\mathrm{CRLB}_{i} x_{i}^{2}}\right. \\
& \left.\times \sum_{i=1}^{N} \frac{\left(l_{t_{i}, 2}-l_{r, 2}\right)^{2}}{\mathrm{CRLB}_{i} x_{i}^{2}}-\left(\sum_{i=1}^{N} \frac{\left(l_{t_{i}, 1}-l_{r, 1}\right)\left(l_{t_{i}, 2}-l_{r, 2}\right)}{\mathrm{CRLB}_{i} x_{i}^{2}}\right)^{2}\right)^{-1} .
\end{aligned}
$$

From (53), the CRLB for position estimation can be specified based on the CRLBs for estimating the distances between the VLC receiver and a number of LED transmitters. Therefore, the results related to distance estimation in Section III provide guidelines for position estimation, as well.

It is important to note that, in the presence of multiple LED transmitters, the VLC receiver can observe and process the signals from the LED transmitters individually by employing multiple access techniques such as time division multiplexing and frequency division multiplexing [12], [14], [31].

\section{CONCLUDING REMARKS}

In this study, theoretical limits and estimators have been obtained for both synchronous and asynchronous VLP systems and in the presence and absence of a relation between distance and channel attenuation factor. In particular, the CRLBs and MLEs have been derived for the TOA based, RSS based, and hybrid TOA/RSS based distance estimation. Comparisons among the CRLBs have been provided, and it has been shown that the CRLB for the hybrid TOA/RSS based estimation converges to that of the TOA based distance estimation for $\beta \gg c / x$, and to that of the RSS based distance estimation for $\beta \ll c / x$. Also, asymptotic results have been obtained for the MLEs under sampling rate limitations, and a modified hybrid TOA/RSS based distance estimator has been proposed to perform accurate distance estimation in practical scenarios. It has been shown that the RSS based and the modified hybrid TOA/RSS based distance estimators can provide robustness against sampling rate limitations, and the modified hybrid TOA/RSS based distance estimator achieves the lowest MSEs among all the estimators in practical scenarios.
As future work, theoretical limits on distance estimation will be considered in the presence of uncertainty about the height of the VLC receiver. In addition, measurements from multiple LED transmitters will be employed to perform hybrid TOA/RSS based estimation in 3-D VLP systems (as outlined below). Another important direction would be to perform an experimental study for evaluating the performance of the MLEs and the tightness of the CRLBs in real-world conditions.

In the presence of multiple LED transmitters, the VLC receiver can process the received signals from the LED transmitters for determining its 3-D position. If $r_{i}(t)$ denotes the received signal from the $i$ th LED transmitter, where $i=1, \ldots, N$, the CRLB expressions and the ML estimators should be derived based on the conditional distribution of $r_{1}(t), \ldots, r_{N}(t)$ given the unknown parameters, which include the location of the VLC receiver and other nuisance parameters, if any. As a practical approach, the VLC receiver can perform two-step position estimation, which has lower implementation complexity and can achieve similar performance to the one-step (joint) optimal processing at high SNRs [27]. In this common approach, position related parameters such as TOA and/or RSS are estimated in the first step and the position of the VLC receiver is estimated based on those position related parameters in the second step. The detailed theoretical analyses and the derivations of the ML estimators and the two step estimators in the presence of multiple LED transmitters are considered as future work.

\section{APPENDIX}

\section{A. Proof of Lemma 1}

Although the proof can be obtained as a special case of the derivation in [21], it is provided below for completeness.

When $\alpha$ is unknown, the vector of unknown parameters becomes $\varphi=(x, \alpha)$ and the log-likelihood function in (5) can be expressed as $\Lambda(x, \alpha)$. Then, the FIM in (6) is given by

$$
\mathbf{J}(x, \alpha)=\left[\begin{array}{ll}
\mathbb{E}\left\{\left(\frac{\partial \Lambda(x, \alpha)}{\partial x}\right)^{2}\right\} & \mathbb{E}\left\{\frac{\partial \Lambda(x, \alpha)}{\partial x} \frac{\partial \Lambda(x, \alpha)}{\partial \alpha}\right\} \\
\mathbb{E}\left\{\frac{\partial \Lambda(x, \alpha)}{\partial \alpha} \frac{\partial \Lambda(x, \alpha)}{\partial x}\right\} & \mathbb{E}\left\{\left(\frac{\partial \Lambda(x, \alpha)}{\partial \alpha}\right)^{2}\right\}
\end{array}\right]
$$

which can be calculated, after some manipulation, as

$$
\mathbf{J}(x, \alpha)=\left(\frac{R_{p}}{\sigma}\right)^{2}\left[\begin{array}{cc}
\alpha^{2} E_{1} / c^{2} & -\alpha E_{3} / c \\
-\alpha E_{3} / c & E_{2}
\end{array}\right]
$$

where $E_{1}, E_{2}$, and $E_{3}$ are given by (10), (13), and (17), respectively. Then, the CRLB on the MSE of any unbiased estimator $\hat{x}$ of $x$ is given by the first element of the inverse of the FIM [24]; that is,

$$
\mathbb{E}\left\{(\hat{x}-x)^{2}\right\} \geq\left[\mathbf{J}(x, \alpha)^{-1}\right]_{1,1}
$$

which can be obtained as in (16) based on (55).

\section{B. Proof of Lemma 2}

For the model in (1), when the TOA parameter $\tau$ is modeled as unknown and the channel attenuation factor $\alpha$ is given by (4), 
the vector of unknown parameters becomes $\varphi=(x, \tau)$ and the log-likelihood function in (5) can be denoted by $\Lambda(x, \tau)$. Then, the FIM in (6) becomes

$$
\mathbf{J}(x, \tau)=\left[\begin{array}{lc}
\mathbb{E}\left\{\left(\frac{\partial \Lambda(x, \tau)}{\partial x}\right)^{2}\right\} & \mathbb{E}\left\{\frac{\partial \Lambda(x, \tau)}{\partial x} \frac{\partial \Lambda(x, \tau)}{\partial \tau}\right\} \\
\mathbb{E}\left\{\frac{\partial \Lambda(x, \tau)}{\partial \tau} \frac{\partial \Lambda(x, \tau)}{\partial x}\right\} & \mathbb{E}\left\{\left(\frac{\partial \Lambda(x, \tau)}{\partial \tau}\right)^{2}\right\}
\end{array}\right] .
$$

The elements of $\mathbf{J}(x, \tau)$ in (57) are obtained, after some manipulation, as

$$
\mathbf{J}(x, \tau)=\left(\frac{\gamma R_{p}}{\sigma}\right)^{2} x^{-2 m-7}\left[\begin{array}{cc}
(m+3)^{2} E_{2} / x & (m+3) E_{3} \\
(m+3) E_{3} & x E_{1}
\end{array}\right]
$$

where $E_{1}, E_{2}$, and $E_{3}$ are given by (10), (13), and (17), respectively. Then, the CRLB on the MSE of any unbiased estimator $\hat{x}$ of $x$ is given by the first element of the inverse of the FIM as stated in (56), which can be obtained as in (30) based on (4) and (58).

\section{REFERENCES}

[1] D. Karunatilaka, F. Zafar, V. Kalavally, and R. Parthiban, "LED based indoor visible light communications: State of the art," IEEE Commun. Surveys Tut., vol. 17, no. 3, pp. 1649-1678, Jul. 2015.

[2] A. Jovicic, J. Li, and T. Richardson, "Visible light communication: Opportunities, challenges and the path to market," IEEE Commun. Mag., vol. 51, no. 12, pp. 26-32, Dec. 2013.

[3] H. Burchardt, N. Serafimovski, D. Tsonev, S. Videv, and H. Haas, "VLC: Beyond point-to-point communication," IEEE Commun. Mag., vol. 52, no. 7, pp. 98-105, Jul. 2014.

[4] A. Sevincer, A. Bhattarai, M. Bilgi, M. Yuksel, and N. Pala, "LIGHTNETs: Smart LIGHTing and mobile optical wireless NETworks-A survey," IEEE Commun. Surveys Tut., vol. 15, no. 4, pp. 1620-1641, Oct. 2013.

[5] J. Armstrong, Y. Sekercioglu, and A. Neild, "Visible light positioning: A roadmap for international standardization," IEEE Commun. Mag., vol. 51, no. 12 , pp. $68-73$, Dec. 2013

[6] T. Wang, Y. Sekercioglu, A. Neild, and J. Armstrong, "Position accuracy of time-of-arrival based ranging using visible light with application in indoor localization systems," J. Lightw. Technol., vol. 31, no. 20, pp. 3302-3308, Oct. 2013.

[7] X. Zhang, J. Duan, Y. Fu, and A. Shi, "Theoretical accuracy analysis of indoor visible light communication positioning system based on received signal strength indicator," J. Lightw. Technol., vol. 32, no. 21, pp. 4180-4186, Nov. 2014

[8] L. Li, P. Hu, C. Peng, G. Shen, and F. Zhao, "Epsilon: A visible light based positioning system," in Proc. 11th USENIX Symp. Netw. Syst. Des. Implementation, Seattle, WA, USA, Apr. 2014, pp. 331-343.

[9] D. Ganti, W. Zhang, and M. Kavehrad, "VLC-based indoor positioning system with tracking capability using Kalman and particle filters," in Proc. IEEE Int. Conf. Consumer Electron., Jan. 2014, pp. 476-477.

[10] Y. Eroglu, I. Guvenc, N. Pala, and M. Yuksel, "AOA-based localization and tracking in multi-element VLC systems," in Proc. IEEE 16th Annu. Wireless Microw. Technol. Conf., Apr. 2015, pp. 1-5.

[11] S.-H. Yang, E.-M. Jung, and S.-K. Han, "Indoor location estimation based on LED visible light communication using multiple optical receivers," IEEE Commun. Lett., vol. 17, no. 9, pp. 1834-1837, Sep. 2013.

[12] Z. Zhou, M. Kavehrad, and P. Deng, "Indoor positioning algorithm using light-emitting diode visible light communications," Opt. Eng., vol. 51, no. 8, pp. 085009-1-085009-7, 2012.

[13] W. Zhang, M. I. S. Chowdhury, and M. Kavehrad, "Asynchronous indoor positioning system based on visible light communications," Opt. Eng., vol. 53, no. 4, pp. 045105-1-045105-9, 2014.

[14] H.-S. Kim, D.-R. Kim, S.-H. Yang, Y.-H. Son, and S.-K. Han, "An indoor visible light communication positioning system using a RF carrier allocation technique," J. Lightw. Technol., vol. 31, no. 1, pp. 134-144, Jan. 2013.
[15] S.-Y. Jung, S. Hann, and C.-S. Park, "TDOA-based optical wireless indoor localization using LED ceiling lamps," IEEE Trans. Consumer Electron., vol. 57, no. 4, pp. 1592-1597, Nov. 2011.

[16] M. Bilgi, A. Sevincer, M. Yuksel, and N. Pala, "Optical wireless localization," Wireless Netw., vol. 18, no. 2, pp. 215-226, Feb. 2012.

[17] S.-H. Yang, H.-S. Kim, Y.-H. Son, and S.-K. Han, "Three-dimensional visible light indoor localization using AOA and RSS with multiple optical receivers," J. Lightw. Technol., vol. 32, no. 14, pp. 2480-2485, Jul. 2014.

[18] A. Hatami and K. Pahlavan, "Hybrid TOA-RSS based localization using neural networks," in Proc. IEEE Global Telecommun. Conf., Nov. 2006, pp. $1-5$.

[19] C. Fritsche and A. Klein, "Cramer-Rao lower bounds for hybrid localization of mobile terminals," in Proc. Workshop Position., Navig. Commun., Mar. 2008, pp. 157-164.

[20] B. Sieskul, T. Kaiser, and F. Zheng, "A hybrid SS-ToA wireless NLoS geolocation based on path attenuation: Cramer-Rao bound," in Proc. IEEE 69th Veh. Technol. Conf., Apr. 2009, pp. 1-5.

[21] S. Gezici, Z. Tian, G. B. Giannakis, H. Kobayashi, A. F. Molisch, H. V. Poor, and Z. Sahinoglu, "Localization via ultra-wideband radios: A look at positioning aspects for future sensor networks," IEEE Signal Process. Mag., vol. 22, no. 4, pp. 70-84, Jul. 2005.

[22] Y. Qi and H. Kobayashi, "Cramér-Rao lower bound for geolocation in non-line-of-sight environment," in Proc. IEEE Int. Conf. Acoust., Speech, Signal Process., May 2002, vol. 3, pp. III-2473-III-2476.

[23] N. Decarli and D. Dardari, "Ziv-Zakai bound for time delay estimation of unknown deterministic signals," in Proc. IEEE Int. Conf. Acoust. Speech Signal Process., May 2014, pp. 4673-4677.

[24] H. V. Poor, An Introduction to Signal Detection and Estimation. New York, NY, USA: Springer-Verlag, 1994.

[25] S. Gezici, H. Celebi, H. Poor, and H. Arslan, "Fundamental limits on time delay estimation in dispersed spectrum cognitive radio systems," IEEE Trans. Wireless Commun., vol. 8, no. 1, pp. 78-83, Jan. 2009.

[26] S. Gezici and Z. Sahinoglu, "Ranging in a single-input multiple-output (SIMO) system," IEEE Commun. Lett., vol. 12, no. 3, pp. 197-199, Mar. 2008.

[27] Z. Sahinoglu, S. Gezici, and I. Guvenc, Ultra-Wideband Positioning Systems: Theoretical Limits, Ranging Algorithms, and Protocols. New York, NY, USA: Cambridge Univ. Press, 2008.

[28] C. Gavrincea, J. Baranda, and P. Henarejos, "Rapid prototyping of standard-compliant visible light communications system," IEEE Commun. Mag., vol. 52, no. 7, pp. 80-87, Jul. 2014.

[29] D. H. Kwon, S. H. Yang, and S. K. Han, "Modulation bandwidth enhancement of white-LED-based visible light communications using electrical equalizations," Proc. SPIE, vol. 9387, pp. 93870T-1-93870T-6, 2015.

[30] Y. Qi, H. Kobayashi, and H. Suda, "On time-of-arrival positioning in a multipath environment," IEEE Trans. Veh. Technol., vol. 55, no. 5, pp. 1516-1526, Sep. 2006.

[31] Y. Hou, S. Xiao, H. Zheng, and W. Hu, "Multiple access scheme based on block encoding time division multiplexing in an indoor positioning system using visible light," IEEE/OSA J. Opt. Commun. Netw., vol. 7, no. 5, pp. 489-495, May 2015.

Musa Furkan Keskin received the B.S. and M.S. degrees both from the Department of Electrical and Electronics Engineering, Bilkent University, Ankara, Turkey, in 2010 and 2012, where he is currently working toward the Ph.D. degree at the same department. His main research interests include the fields of statistical signal processing, wireless communications, and visible light positioning.

Sinan Gezici received the B.S. degree from Bilkent University, Ankara, Turkey, in 2001, and the Ph.D. degree in Electrical Engineering from Princeton University, Princeton, NJ, USA, in 2006.

From 2006 to 2007, he was with Mitsubishi Electric Research Laboratories, Cambridge, MA, USA. Since 2007, he has been at the Department of Electrical and Electronics Engineering, Bilkent University, where he is currently an Associate Professor. His research interests include the areas of detection and estimation theory, wireless communications, and localization systems. Among his publications in these areas, he has published the book Ultra-wideband Positioning Systems: Theoretical Limits, Ranging Algorithms, and Protocols (Cambridge, U.K.: Cambridge Univ. Press, 2008). He is an Associate Editor for the IEEE TRANSACTIONS ON COMMUNICATIONS, the IEEE WIRELESS COMMUNICATIONS LETTERS, and the Journal of Communications and Networks. 